\title{
Relation of pupillary light reflex to light intensity
} Antonis Tzambazakis*, Dimitrios Fotiou, Aggelis Fotiou, Dimitrios Tsiptsios and Maria Nakou

\author{
Address: Laboratory of Clinical Neurophysiology, Aristotle University of Thessaloniki, Greece \\ * Corresponding author
}

from International Society on Brain and Behaviour: 2nd International Congress on Brain and Behaviour Thessaloniki, Greece. 17-20 November 2005

Published: 28 February 2006

Annals of General Psychiatry 2006, 5(SuppI I):S330 doi:I0.I 186/I744-859X-5-SI-S330

\section{Background}

This study was carried out to investigate the effect of light intensity of the flash stimulus on the pupillary light reflex (PLR) in normal healthy volunteers.

\section{Materials and methods}

The pupillary recording system used is computer controlled, includes an infrared digital video camera capable of recording 260 frames/sec $(260 \mathrm{~Hz})$ and it provides real time measurements, online full statistical analysis of the results and averaging of the important parameters. Pupil reactions of twenty normal healthy volunteers aged 20 to 28 were measured for 7 light intensity levels of the flash. Stimulus parameters were flash light wavelength $555 \mathrm{~nm}$, retinal illuminance intensity-1230 trolands, 150 trolands, 100 trolands, 25 trolands, 6.4 trolands, 1.6 trolands and 0.1 trolands - stimulus time $10 \mathrm{~ns}$, background retinal illumination 0 trolands and interstimulus interval $3 \mathrm{~s}$. Pupillometric parameters studied were reaction time, initial Radius, minimum Radius, min/initial Radius [\%], final/initial Radius [\%], time for maximum myosis, maximum velocity and maximum acceleration.

\section{Results}

The measured range of retinal illuminance levels (0.11230 trolands) of the flash stimulus didn't affect significantly the pupillary light reflex until the retinal illuminance approached the 150 trolands for $5(25 \%)$ of the subjects, 100 trolands for 9 (45\%) of the subjects and 25 trolands for $6(30 \%)$ of the subjects. From these thresholds and below there was an induce of the minimum radius and of the reaction time and a reduce of the time for maximum myosis, of maximum velocity and of maximum acceleration.

\section{Discussion}

The results from this study suggest that for a wide range of light intensity levels of the flash stimulus, the pupillary light reflex is not affected significantly. But at the level of 150 trolands and below we have significant changes to the minimum radius, reaction time, time for maximum velocity and maximum acceleration. 